\title{
Functional Impairment Is a Risk Factor for Knee Replacement in the Multicenter Osteoarthritis Study
}

\author{
Barton L. Wise MD, MSc, Jingbo Niu DSc, David T. Felson MD, MPH, \\ Jean Hietpas LCSW, Alesia Sadosky PhD, MPH, MBA, James Torner PhD, MS, \\ Cora E. Lewis MD, MSPH, Michael Nevitt PhD, MPH
}

Published online: 10 March 2015

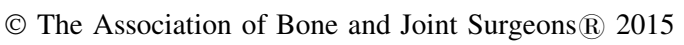

\begin{abstract}
Background Debilitating pain associated with knee osteoarthritis (OA) often leads patients to seek and complete total knee arthroplasty (TKA). To date, few studies have evaluated the relation of functional impairment to the risk of TKA, despite the fact that OA is associated with functional impairment.

Questions/purposes The purpose of our study was to (1) evaluate whether function as measured by WOMAC
\end{abstract}

Funded by National Institutes of Health (NIH) grants UO1 AG18820 (TF), UO1 AG18832 (JT), UO1 AG18947 (CEL), and UO1 AG19069 (MN); additional funding for this work came from NIH grant NICHD RO1 HD43500. This work was also supported by the following funding sources: the Center for Musculoskeletal Health at University of California, Davis School of Medicine, NIH K24 AR048841 (NEL), NIH P50 AR060752 (BLW, NEL), NIH P50 AR063043 (BLW,

NEL), the University of California, Davis Building Interdisciplinary Research Careers in Women's Health Program (NIH K12HD051958) (BLW), and the Endowed Chair for Aging at University of California, Davis School of Medicine (NEL). One of the authors (BLW) received research funding from Pfizer Inc for this analysis through the University of California, Davis in the amount of USD 100,001 to USD 1,000,000. One of the authors (AS) is an employee of Pfizer Inc (New York, NY, USA).

All ICMJE Conflict of Interest Forms for authors and Clinical Orthopaedics and Related Research ${ }^{\circledR}$ editors and board members are on file with the publication and can be viewed on request.

Each author certifies that his or her institution approved or waived approval for the reporting of this study and that all investigations were conducted in conformity with ethical principles of research. Information was collected from participants in the Multicenter Osteoarthritis Study at the two clinical centers (University of Alabaman, Birmingham, AL, USA, and University of Iowa, Iowa City, IA, USA). Conception, design, and implementation of the analysis were performed at University of California, Davis, University of California, San Francisco, CA, USA, and Boston physical function subscale was associated with undergoing TKA; and (2) whether any such association varied by sex. Methods The National Institutes of Health-funded Multicenter Osteoarthritis Study (MOST) is an observational cohort study of persons aged 50 to 79 years with or at high risk of symptomatic knee OA who were recruited from the community. All eligible subjects with complete data were included in this analysis. Our study population sample consisted of 2946 patients with 5796 knees; 1776 (60\%) of

University, Boston, MA, USA. Statistical analysis was performed at Boston University, Boston, MA, USA.

\section{B. L. Wise $(\square)$}

Center for Musculoskeletal Health, University of California, Davis School of Medicine, 4625 2nd Avenue, Suite 2000,

Sacramento, CA 95817, USA

e-mail: barton.wise@ucdmc.ucdavis.edu

\section{J. Niu, D. T. Felson}

Clinical Epidemiology Research and Training Unit, Boston

University School of Medicine, Boston, MA, USA

\section{J. Hietpas, M. Nevitt}

Department of Epidemiology and Biostatistics, University of California, San Francisco, San Francisco, CA, USA

A. Sadosky

Pfizer Inc, New York, NY, USA

J. Torner

Department of Epidemiology, University of Iowa College of Public Health, Iowa City, IA, USA

\section{E. Lewis}

Birmingham School of Medicine, University of Alabama, Birmingham, AL, USA 
patients were women. We performed a repeated-measures analysis using baseline WOMAC physical function score to predict the risk of TKA from baseline to 30 months and WOMAC score at 30 months to predict risk of incident TKA from 30 months to 60 months. We used generalized estimating equations to account for the correlation between two knees within an individual and across the two periods. We calculated relative risk (RR) of TKA over 30 months by WOMAC function using a score of 0 to 5 as the referent in multiple binomial regressions with log link.

Results Those with the greatest functional impairment (WOMAC scores 40-68; 62 TKAs in 462 knee periods) had 15.5 times (95\% confidence interval [CI], 7.6-31.8; $\mathrm{p}<0.001)$ the risk of undergoing TKA over 30 months compared with the referent group (12 TKAs in 3604 knee periods), adjusting for basic covariates, and 5.9 times (95\% CI, 2.8-12.5; $\mathrm{p}<0.001)$ the risk after further adjusting for knee pain severity. At every level of functional limitation, the RR for TKA for women was higher than for men, but interaction with sex did not reach significance after adjustment for covariates including ipsilateral pain $(\mathrm{p}=0.138)$.

Conclusions Baseline physical function appears to be an important element in patients considering TKA. Future studies should examine whether interventions to improve function can reduce the need for TKA.

Level of Evidence Level III, observational cohort study.

\section{Introduction}

Osteoarthritis (OA) is the most common form of arthritis and the leading cause of disability in the elderly. Knee arthroplasty is the most effective treatment for patients with severe OA of the knee. A large proportion of the billions of healthcare dollars spent each year in the United States for OA is directed either toward joint replacement or toward alleviating functional impairment resulting from OA [10]. The amount of societal investment in treating OA has been increasing and is certain to grow substantially during the next 10 to 20 years as the population ages and obesity (a risk factor for OA) continues to become more prevalent.

The most definitive treatment for knee pain associated with advanced knee OA is TKA. In general, the decision to replace a knee is based on patient severity of pain and limitations of function, and surgeons work with their patients to help them make knee arthroplasty decisions. As Dieppe et al. report, "there are no evidence-based indications for total knee replacement in knee OA" [3]. In a postal survey of orthopaedists, most reported that patients with severe daily pain with radiographic joint space narrowing were the most common reason they replaced knees [11]. Race, age, and sex have all been identified as affecting the likelihood of a particular patient receiving a joint replacement [6] and thus likely confounding the relation between pain and knee replacement. Functional impairment may influence an individual to undergo TKA but few studies have explored this assumption.

Previously, studies have found that a substantial proportion of TKAs are performed in patients who on radiography appeared to have only mild or moderate knee OA $[9,12,14]$. Furthermore, only a portion of patients with severe radiographic knee OA end up seeking or undergoing TKA. Other pathological manifestations that are not identified by radiography may play a role in the rate of OA progression and subsequent TKA. Severity of knee pain is known to predict incident TKA in study subjects with OA, and our group has recently identified other dimensions of pain as increasing the likelihood of TKA. Patients with knee OA have been identified as having reduced muscle function and functional capacity compared with control subjects [5]. To date, few studies to our knowledge have examined the relation of functional impairment to risk of joint replacement.

The purpose of our study was to (1) evaluate whether function as measured by the WOMAC physical function subscale was associated with having a TKA; and (2) whether any such association varied by sex.

\section{Materials and Methods}

The Multicenter Osteoarthritis (MOST) Study is a National Institutes of Health-funded observational cohort study, which enrolled persons recruited from the community aged between 50 and 79 years at baseline who either had symptomatic knee OA or were at high risk of the disease [4]. We conducted a cohort study to evaluate the association between functional impairment as measured by WOMAC [1] physical function score with the risk of TKA over 30 months; WOMAC physical function was measured with Likert scales in different domains of function with a total range from 0 to 68 with 0 representing no difficulty with functional activities and 68 signifying extreme difficulty with activities. Our endpoint was first TKA in a knee during the study; TKA was reported by patients. WOMAC function measurement at baseline and at 30 months was used as a predictor (study visits in the MOST study were conducted at baseline, 30 months, and 60 months). We performed a repeatedmeasures analysis. Baseline WOMAC score was used to predict the risk of TKA over baseline to 30 months, whereas WOMAC score at 30 months was used to predict the incident TKA from 30 months to 60 months. A knee period refers to a single knee in a single 30 -month period $(0-30$ months or 30-60 months). Thus, in this study, each participant may potentially contribute up to four knee periods (two knees in two periods). 
Table 1. Flowchart showing losses to followup

\begin{tabular}{|c|c|}
\hline Subjects followed from baseline to 30 -month visit (number) & Reason for loss to followup or exclusion from analysis \\
\hline 3026 & Recruited at baseline \\
\hline-3 & Rheumatoid arthritis \\
\hline-20 & Baseline WOMAC function missing \\
\hline-29 & Death before 30 -month visit \\
\hline-5 & Withdrew consent by 30 -month visit \\
\hline-23 & Telephone interview not done for any reason at 30-month visi \\
\hline 2946 & Used for 0 - to 30 -month period \\
\hline \multicolumn{2}{|l|}{ Subjects followed from 30- to 60 -month visit (number) } \\
\hline 2966 & Non-RA subjects with 30-month visit telephone interview \\
\hline-43 & Bilateral TKA before 30-month visit \\
\hline-270 & WOMAC function missing at 30-month visit \\
\hline-23 & $\begin{array}{l}\text { 30-month visit knee radiographs not done or radiograph reading } \\
\text { excluded as a result of osteonecrosis, etc }\end{array}$ \\
\hline-48 & Death before 60 -month visit \\
\hline-23 & Withdrew consent by 60 -month visit \\
\hline-5 & Discontinued by 60 -month visit \\
\hline-59 & Telephone interview not done for any reason at 60 -month visit \\
\hline 2495 & Used for 30 - to 60 -month period \\
\hline
\end{tabular}

$\mathrm{RA}=$ rheumatoid arthritis.

Plain knee radiographs were performed at baseline and at 30 months with weightbearing fixed flexion in-frame posteroanterior radiographs read for Kellgren/Lawrence $(\mathrm{K} / \mathrm{L})$ grade by two readers with disagreements subsequently adjudicated by a separate panel reviewing the film. We eliminated patients with TKA in both knees at baseline from the analysis. Knees with radiologic signs of fracture, malignancy, osteonecrosis, and trauma were excluded from all of our analyses. Knees or patients with missing information relevant to our study at baseline or at 30 or 60 months were excluded as were those with rheumatoid arthritis (Table 1). Other than subjects with these described exclusions, all subjects were included in the analysis. Baseline and followup visits included telephone interviews, radiographic studies, face-to-face interviews, and clinical in-person assessment, all as part of the MOST study and separate from any clinical care that the subjects might receive from their physicians.

The MOST study enrolled 3026 participants at baseline. After patients and knees were eliminated as a result of TKA being present at baseline, rheumatoid arthritis, missing information, and other reasons described previously, our sample consisted of 2946 patients with 5796 knees ( 72 knees were excluded as a result of TKA before recruitment; a further 24 knees were eliminated as a result of radiograph reading problems); $1776(60 \%)$ patients were women. Of the 2946 patients, 1263 (43\%) had a baseline
WOMAC function score less than 10 (range, 0-68). Over 60 months, there were 462 incident TKAs in 10,629 knee periods (4.4\%). Of the 462 incident TKAs, 327 (71\%) occurred in women and $195(42 \%)$ occurred in the first 30-month period.

We first used graphical and tabular descriptive statistics to check for the presence of outliers of all risk factors and potential confounders. We compared the characteristics of functionally impaired and nonimpaired knees as to their distribution of age, sex, ethnicity, study center, education, occupation, body mass index (BMI), history of knee injury, and comorbidities assessed at baseline visit. We calculated the incidence of TKA in men and women over 60 months.

We calculated the risk of undergoing TKA over 30 months according to status of functional impairment. We examined the association between function and risk of TKA using generalized estimating equations to account for the correlation between two knees within one subject and across the two periods of the repeated analysis. We calculated relative risk (RR) of undergoing TKA for groupings of WOMAC function 0 to 5,6 to 9,10 to 19,20 to 29,30 to 39 , and 40 to 68 using the 0 to 5 group as the referent. In the multiple regression model, we adjusted for clinic site, age, BMI, race, education, depressive symptoms as measured by Center for Epidemiologic Studies Depression Scale (CES-D), comorbidity, ipsilateral K/L grade, and, in the combined gender analysis, we also 
Table 2. Association of WOMAC function with incident TKA over 30 months, men and women combined

\begin{tabular}{|c|c|c|c|c|c|c|c|c|}
\hline \multirow[t]{2}{*}{$\begin{array}{l}\text { WOMAC function } \\
(0-68)\end{array}$} & \multirow[t]{2}{*}{$\begin{array}{l}\text { Knee periods } \\
\text { (number) }\end{array}$} & \multirow[t]{2}{*}{$\begin{array}{l}\text { Number of TKAs } \\
(\%)\end{array}$} & \multicolumn{2}{|l|}{ Crude model } & \multicolumn{2}{|c|}{$\begin{array}{l}\text { Adjusted model (basic } \\
\text { covariates*) }\end{array}$} & \multicolumn{2}{|c|}{$\begin{array}{l}\text { Adjusted model (basic } \\
\text { covariates plus } \\
\text { ipsilateral VAS pain } \\
\text { score) }\end{array}$} \\
\hline & & & RR (95\% CI) & $\mathrm{p}$ value & RR $(95 \% \mathrm{CI})$ & $\mathrm{p}$ value & $\mathrm{RR}(95 \% \mathrm{CI})$ & $\mathrm{p}$ value \\
\hline $0-5$ & 3604 & $12(0.33)$ & 1.0 & & 1.0 & & 1.0 & \\
\hline $6-9$ & 1174 & $20(1.70)$ & $5.2(2.3-11.8)$ & $<0.001$ & $3.5(1.6-7.9)$ & 0.002 & $3.1(1.4-7.0)$ & 0.006 \\
\hline $10-19$ & 2439 & $98(4.02)$ & $11.6(5.8-23.1)$ & $<0.001$ & $5.6(2.8-11.2)$ & $<0.001$ & $4.4(2.2-8.8)$ & $<0.001$ \\
\hline $20-29$ & 1870 & $145(7.75)$ & $22.2(11.3-43.8)$ & $<0.001$ & $8.7(4.4-17.1)$ & $<0.001$ & $5.4(2.7-10.8)$ & $<0.001$ \\
\hline $30-39$ & 1080 & $125(11.57)$ & $33.4(16.9-65.9)$ & $<0.001$ & $11.8(5.9-23.4)$ & $<0.001$ & $5.9(2.9-12.0)$ & $<0.001$ \\
\hline $40-68$ & 462 & $62(13.42)$ & $39.1(19.3-79.2)$ & $<0.001$ & $15.5(7.6-31.8)$ & $<0.001$ & $5.9(2.8-12.5)$ & $<0.001$ \\
\hline $\mathrm{p}$ for linear trend & & & & & & $<0.001$ & & $<0.001$ \\
\hline
\end{tabular}

* Clinic site, age, body mass index, race, education, Center for Epidemiologic Studies Depression Scale, comorbidity, ipsilateral KellgrenLawrence grade, sex; VAS $=$ visual analog scale; $\mathrm{RR}=$ relative risk.

Table 3. Association of WOMAC function with incident TKA over 30 months, men and women, separately (no adjustment for ipsilateral pain)

\begin{tabular}{|c|c|c|c|c|c|c|c|c|}
\hline \multirow{2}{*}{$\begin{array}{l}\text { WOMAC } \\
\text { function } \\
(0-68)\end{array}$} & \multicolumn{4}{|l|}{ Women } & \multicolumn{4}{|l|}{ Men } \\
\hline & $\begin{array}{l}\text { Knee periods } \\
\text { (number) }\end{array}$ & $\begin{array}{l}\text { Number of } \\
\text { TKAs }(\%)\end{array}$ & $\begin{array}{l}\text { Adjusted RR }(95 \% \text { CI) } \\
\text { (basic covariates*) }\end{array}$ & $\mathrm{p}$ value & $\begin{array}{l}\text { Knee periods } \\
\text { (number) }\end{array}$ & $\begin{array}{l}\text { Number of } \\
\text { TKAs }(\%)\end{array}$ & $\begin{array}{l}\text { Adjusted RR }(95 \% \text { CI) } \\
\text { (basic covariates*) }\end{array}$ & $\mathrm{p}$ value \\
\hline $0-5$ & 1863 & $6(0.32)$ & 1.0 & & 1741 & $6(0.34)$ & 1.0 & \\
\hline $6-9$ & 692 & $13(1.88)$ & $4.3(1.4-13.0)$ & 0.01 & 482 & $7(1.45)$ & $2.8(0.9-9.1)$ & 0.084 \\
\hline $10-19$ & 1515 & $63(4.16)$ & $6.3(2.4-16.4)$ & $<0.001$ & 924 & 35 (3.79) & $5.0(1.8-13.7)$ & 0.002 \\
\hline $20-29$ & 1227 & $108(8.80)$ & $9.9(3.8-25.6)$ & $<0.001$ & 643 & $37(5.75)$ & $7.3(2.7-19.7)$ & $<0.001$ \\
\hline $30-39$ & 760 & 89 (11.71) & $12.1(4.7-31.6)$ & $<0.001$ & 320 & $36(11.25)$ & $13.3(5.0-35.6)$ & $<0.001$ \\
\hline $40-68$ & 324 & $48(14.81)$ & $16.8(6.2-45.3)$ & $<0.001$ & 138 & $14(10.14)$ & $15.8(5.4-46.5)$ & $<0.001$ \\
\hline $\begin{array}{l}\mathrm{p} \text { for linear } \\
\text { trend }\end{array}$ & & & & $<0.001$ & & & & $<0.001$ \\
\hline
\end{tabular}

* Clinic site, age, body mass index, race, education, Center for Epidemiologic Studies Depression Scale, comorbidity, ipsilateral KellgrenLawrence grade; $\mathrm{RR}=$ relative risk.

adjusted for sex. We also performed analyses both with and without adjustment for ipsilateral visual analog scale (VAS; range, 0-100) knee pain to assess whether an association between physical function or functional impairment and TKA was independent of pain. We tested for potential effect modification by sex by adding an interaction term between gender and WOMAC function to the regression model. Finally, we generated receiver operating characteristic (ROC) curves and associated C statistics (or area under the ROC curve) [2] to illustrate the predictability of risk of undergoing TKA from physical function score. All analyses were done using SAS Version 9.2 (SAS Institute Inc, Cary, NC, USA) and analyses performed at the $5 \%$ significance level; for all analyses, one of the authors ( JN) acted as the programmer, and all authors had input into the approach and interpretation.

\section{Results}

Patients with the highest function scores (greatest functional impairment; 40-68) had 15.5 times the risk of TKA over 30 months compared with those with baseline function scores of 0 to 5 after adjustment for covariates except pain $(95 \%$ confidence interval [CI], 7.6-31.8; $\mathrm{p}$ for linear trend $<0.001$; Table 2). Further adjustment for ipsilateral knee pain attenuated the effect of function score on the risk of undergoing TKA, especially among those with the worst function score, but the trend of association was still significant ( $\mathrm{p}$ for linear trend $<0.001$ ).

WOMAC function was associated with incident TKA in both men and women, separately at every stratum, except for those men with WOMAC of 6 to 9, where significance was not achieved in the adjusted analyses. Also, at every 
level, the RR for women was higher than for men (Table 3). Interaction by sex for the model adjusted for basic covariates plus ipsilateral pain had a $\mathrm{p}$ for interaction equal to 0.138; when the model was adjusted only for basic covariates, the $\mathrm{p}$ value generated for interaction was 0.240 .

The WOMAC function score explained a substantial amount of the risk for undergoing TKA in the ROC curves we generated. The $\mathrm{C}$ statistic was 0.80 for men only, 0.76 for women only, and 0.78 for men and women together (ROC curve for both sexes is shown in Fig. 1).

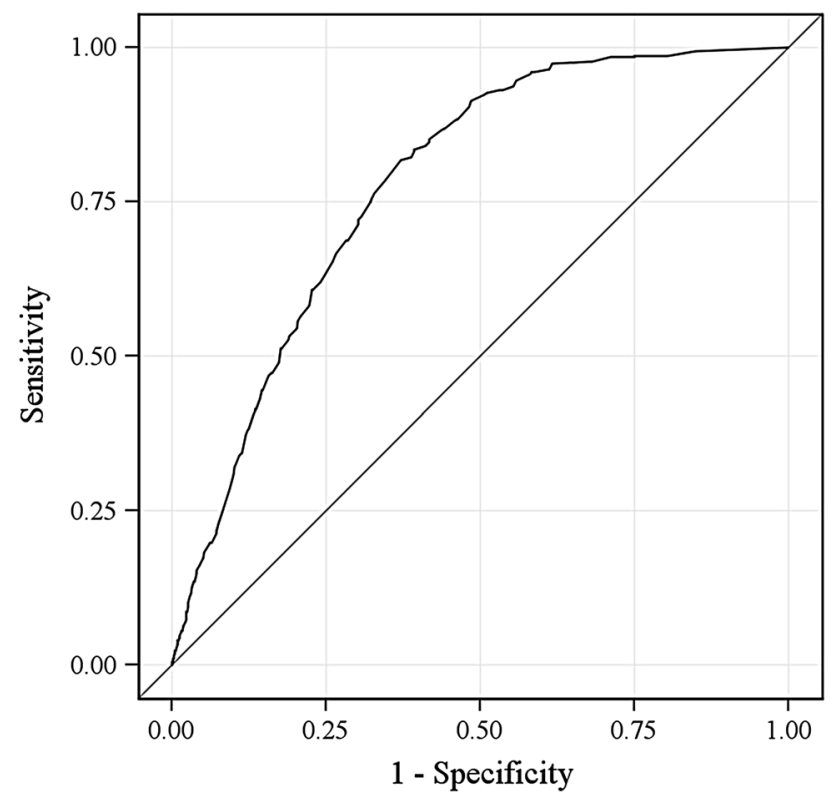

Fig. 1 ROC curve for men and women combined demonstrates that functional impairment explains a significant amount of the risk for undergoing TKA.
Patients who underwent incident TKA during the 60 months were slightly older (mean age 65 versus 62 years) and heavier (mean BMI 33 versus $31 \mathrm{~kg} / \mathrm{m}^{2}$ ) than subjects who did not receive TKA; $71 \%$ were women compared with $59 \%$ of those without TKA. Sixty-two percent of those with TKA had some college education compared with $73 \%$ of those with no TKA. They had more severe radiographic knee OA compared with those without TKA (Table 4).

Patients with higher WOMAC function categories (greater functional impairment) were older, had higher BMI, were more likely to be women, more likely to be nonwhite, had lower educational attainment, had greater VAS pain, had higher CES-D scores, and higher comorbidity scores (Table 5).

\section{Discussion}

OA with radiographic changes and severe pain is frequently treated with TKA. We investigated the understudied question of whether functional limitation predisposed subjects to completing a TKA and whether there are differences by sex in the association. We found a strong predictive association between functional impairment and subsequent TKA, and this relationship is independent of pain. This relationship holds true both for relatively mild functional impairment and for severe impairment with a very impressive and appropriate doseresponse. We were unable to identify a significant interaction with sex for the association of functional impairment with TKA.

There are limitations to our analysis. First, there was not a fixed period of time between ascertainment of functional

Table 4. Characteristics of subjects by incident TKA status between baseline and 60 months

\begin{tabular}{|c|c|c|c|}
\hline Characteristic & Without TKA ( $\mathrm{n}=2597$ patients) & With TKA ( $\mathrm{n}=349$ patients) & $\mathrm{p}$ value \\
\hline Age (mean years; $\pm \mathrm{SD}$ ) & $62( \pm 8.1)$ & $65( \pm 7.4)$ & $<0.001$ \\
\hline $\mathrm{BMI}\left(\right.$ mean $\left.\mathrm{kg} / \mathrm{m}^{2} ; \pm \mathrm{SD}\right)$ & $31( \pm 5.8)$ & $33( \pm 6.6)$ & $<0.001$ \\
\hline Sex $(\%)$ : women & $1530(59)$ & $246(71)$ & $<0.001$ \\
\hline Race $(\%)$ : white & $2160(83)$ & 307 (88) & 0.022 \\
\hline College (\%): yes & $1882(73)$ & $216(62)$ & $<0.001$ \\
\hline Maximum VAS pain, mean $( \pm$ SD) & $23( \pm 23)$ & $43( \pm 24)$ & $<0.001$ \\
\hline CES-D, mean $( \pm \mathrm{SD})$ & $7.4( \pm 7.6)$ & $8.2( \pm 8.2)$ & 0.061 \\
\hline Comorbidity, mean number ( \pm SD) & $0.5( \pm 0.9)$ & $0.6( \pm 0.9)$ & 0.079 \\
\hline Kellgren-Lawrence grade $(\%)$ & & & $<0.001$ \\
\hline 0 & $992(38.2)$ & $9(2.6)$ & \\
\hline 1 & $471(18.1)$ & $11(3.2)$ & \\
\hline 2 & $452(17.4)$ & $31(8.9)$ & \\
\hline $3-4$ & $682(26.3)$ & $298(85.4)$ & \\
\hline
\end{tabular}

BMI = body mass index; VAS = visual analog scale; CES-D = Center for Epidemiologic Studies Depression Scale. 
2510 Wise et al.

Clinical Orthopaedics and Related Research ${ }^{\circledR}$

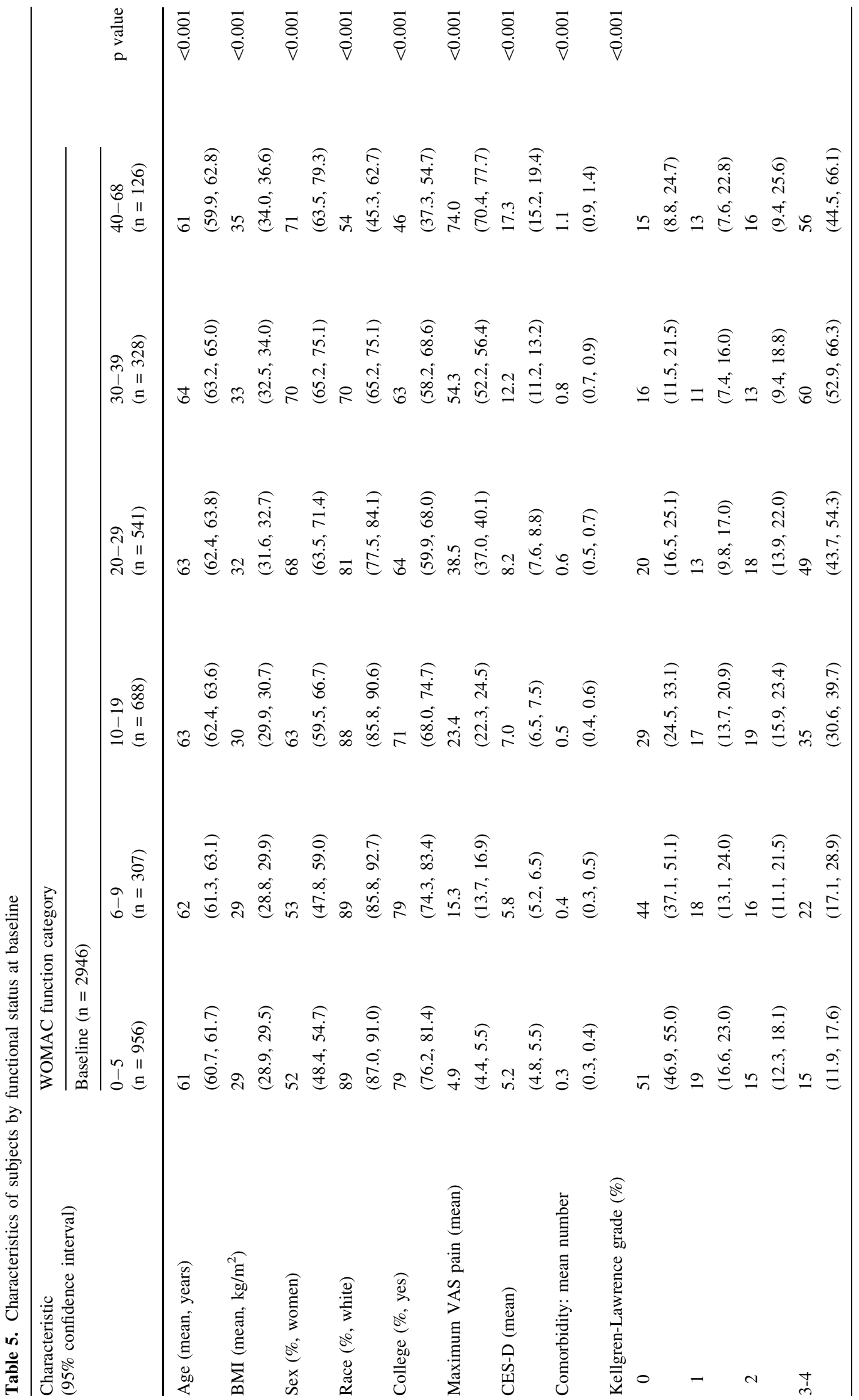

Springer 


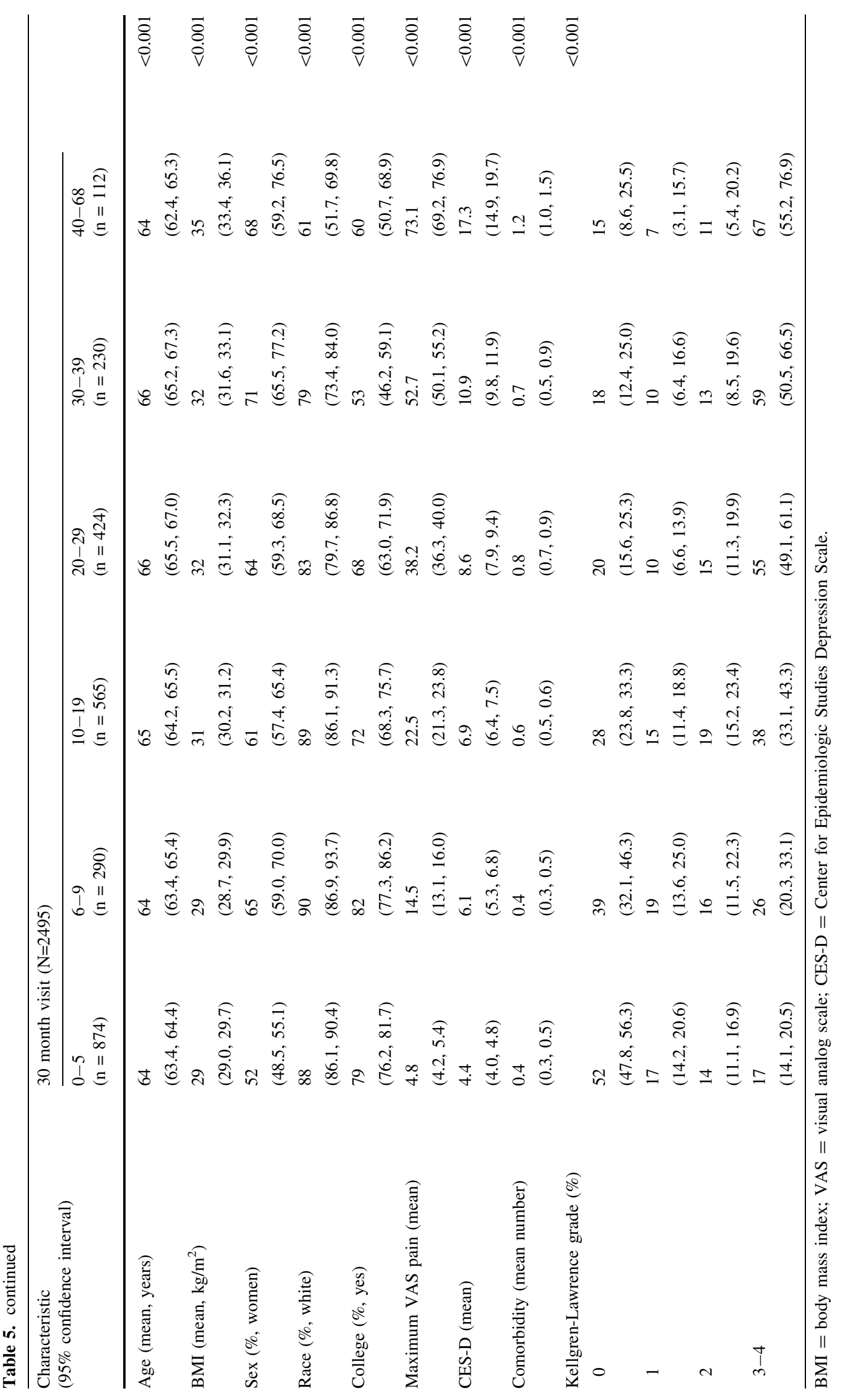


status and TKA. We tried to minimize the bias deriving from this limitation by splitting the period of observation into two knee periods of 30 months each. This limited the maximum delay between function ascertainment and TKA. We note that Kelly et al. reported there was no significant difference in decline of WOMAC function between patients waiting 1 to 3 months, 3 to 6 months, and greater than 6 months for TKA [8]; of course, 30 months is substantially longer than the 6 months evaluated in the Kelly et al. study but nonetheless, it provides some evidence that supports the validity of the timing of our study. Second, the study population in MOST was aged between 50 and 79 years at baseline and the patients either had knee OA or were at risk of the disease, so our results may not be generalizable to a younger population. Third, because we used all eligible subjects and only a minimal proportion of the subjects were not used (because of missing information, death, etc), we feel that selection bias would be small and unable to explain the large effect sizes we identified. Fourth, although we controlled for many important confounders, residual confounding for the covariates as well as unadjusted confounders may remain.

There were also significant strengths to our study. First, the MOST cohort is large and loss to followup was relatively small over the period of 60 months. The study variables were carefully chosen, well characterized, and collected meticulously at every time point. Radiographs in MOST were read centrally by expert readers and the readings have been evaluated for reliability and used for many other studies.

Ours is the first study to our knowledge to examine the contribution of functional status to risk of undergoing TKA in a prospective fashion. As it turns out, this contribution is quite significant as attested to both by the magnitude of the effect sizes observed as well as by the C statistic of 0.78 . Thorstensson et al. examined the contribution of reduction of lower extremity functional performance to incidence or progression of radiographic knee OA in a group of Swedish patients [16]. They used objective measures of functional performance (time spent walking $300 \mathrm{~m}$, timed standing on one leg, and maximum number of one-leg rises from sitting), and reported that only maximum number of one-leg raises was significantly associated with incident $\mathrm{OA}$ over 5 years, whereas none of the measures was associated with progression of radiographic OA during the same time period. Thus, subgroups of subjects both with and without $\mathrm{OA}$ at baseline (similar to our study) were examined with mixed results. Our study had a different outcome, TKA, one that is related conceptually to progression and to incident radiographic OA. That we found such a strong association compared with the relatively weaker association identified by Thorstensson et al. may be the result of multiple reasons, including our use of the WOMAC (a subjective measure of function), our outcome of TKA, and differences in the study populations (the Swedish study included younger patients than patients in MOST, with chronic knee pain, who were selected randomly from an urban and rural population).

Interestingly, the association of function with TKA appeared to be stronger for women than for men, although the interaction term did not reach significance, likely as a result of small sample size. It is possible that sex differences may be the result of mechanical influences [13]. Katz et al. reported significant differences by sex in functional status before TKA and noted that the differences were not the result of clinical characteristics of the patient, suggesting that patient preferences and access to surgery were possible reasons [7]. van Dijk et al. reported no prognostic association between sex and future functional status over 3 years of followup in patients with knee OA [17]. It was reported that physical inactivity was associated with severe joint pain among men but not women [15], suggesting that there may be a complex relationship among activity levels, functional impairment, and pain, which may differ by sex.

In summary, baseline physical functional impairment is an important element in patients considering TKA. Future studies should examine whether interventions to improve function can reduce the need for TKA.

Acknowledgments We thank Yuqing Zhang and Nancy E. Lane for advice and mentoring during the course of our study analyses. We also thank the MOST participants.

\section{References}

1. Bellamy N, Buchanan WW, Goldsmith $\mathrm{CH}$, Campbell J, Stitt LW. Validation study of WOMAC: a health status instrument for measuring clinically important patient relevant outcomes to antirheumatic drug therapy in patients with osteoarthritis of the hip or knee. J Rheumatol. 1988;15:1833-1840.

2. Cook NR. Use and misuse of the receiver operating characteristic curve in risk prediction. Circulation. 2007;115:928-935.

3. Dieppe P, Basler HD, Chard J, Croft P, Dixon J, Hurley M, Lohmander S, Raspe H. Knee replacement surgery for osteoarthritis: effectiveness, practice variations, indications and possible determinants of utilization. Rheumatology (Oxford). 1999;38:73-83.

4. Felson DT, Nevitt MC. Epidemiologic studies for osteoarthritis: new versus conventional study design approaches. Rheum Dis Clin North Am. 2004;30:783-797, vii.

5. Fisher NM, Pendergast DR. Reduced muscle function in patients with osteoarthritis. Scand J Rehabil Med. 1997;29:213-221.

6. Katz BP, Freund DA, Heck DA, Dittus RS, Paul JE, Wright J, Coyte P, Holleman E, Hawker G. Demographic variation in the rate of knee replacement: a multi-year analysis. Health Serv Res. 1996;31:125-140.

7. Katz JN, Wright EA, Guadagnoli E, Liang MH, Karlson EW, Cleary PD. Differences between men and women undergoing major orthopedic surgery for degenerative arthritis. Arthritis Rheum. 1994;37:687-694. 
8. Kelly KD, Voaklander DC, Johnston DW, Newman SC, SuarezAlmazor ME. Change in pain and function while waiting for major joint arthroplasty. J Arthroplasty. 2001;16:351-359.

9. Keurentjes JC, Fiocco M, So-Osman C, Onstenk R, KoopmanVan Gemert AW, Poll RG, Kroon HM, Vliet Vlieland TP, Nelissen RG. Patients with severe radiographic osteoarthritis have a better prognosis in physical functioning after hip and knee replacement: a cohort-study. PloS One. 2013;8:e59500.

10. Kim S. Changes in surgical loads and economic burden of hip and knee replacements in the US: 1997-2004. Arthritis Rheum. 2008; 59:481-488.

11. Mancuso CA, Ranawat CS, Esdaile JM, Johanson NA, Charlson ME. Indications for total hip and total knee arthroplasties. Results of orthopaedic surveys. J Arthroplasty. 1996;11:34-46.

12. Meding JB, Ritter MA, Faris PM, Keating EM, Harris W. Does the preoperative radiographic degree of osteoarthritis correlate to results in primary total knee arthroplasty? J Arthroplasty. 2001; $16: 13-16$

13. Nicolella DP, O'Connor MI, Enoka RM, Boyan BD, Hart DA, Resnick E, Berkley KJ, Sluka KA, Kwoh CK, Tosi LL, Coutts
RD, Havill LM, Kohrt WM. Mechanical contributors to sex differences in idiopathic knee osteoarthritis. Biol Sex Differ. 2012;3:28.

14. Polkowski GG 2nd, Ruh EL, Barrack TN, Nunley RM, Barrack RL. Is pain and dissatisfaction after TKA related to early-grade preoperative osteoarthritis? Clin Orthop Relat Res. 2013;471: $162-168$.

15. Shih M, Hootman JM, Kruger J, Helmick CG. Physical activity in men and women with arthritis National Health Interview Survey, 2002. Am J Prev Med. 2006;30:385-393.

16. Thorstensson CA, Petersson IF, Jacobsson LT, Boegard TL, Roos EM. Reduced functional performance in the lower extremity predicted radiographic knee osteoarthritis five years later. Ann Rheum Dis. 2004;63:402-407.

17. van Dijk GM, Dekker J, Veenhof C, van den Ende CH. Course of functional status and pain in osteoarthritis of the hip or knee: a systematic review of the literature. Arthritis Rheum. 2006;55: 779-785. 